\title{
Singular Perturbation Analysis of Travelling Waves for a Model in Phytopathology
}

\author{
J.B. Burie ${ }^{a}$, A. Calonnec ${ }^{b}$ and A. Ducrot ${ }^{a 1}$ \\ ${ }^{a}$ UMR CNRS 5466, MAB \& INRIA Futurs, Université Victor Segalen Bordeaux 2 \\ 33076 Bordeaux, France \\ ${ }^{b}$ INRA-CR de Bordeaux, UMR INRA-ENITA en Santé végétale, 33883 Villenave d'Ornon
}

\begin{abstract}
We investigate the structure of travelling waves for a model of a fungal disease propagating over a vineyard. This model is based on a set of ODEs of the SIR-type coupled with two reaction-diffusion equations describing the dispersal of the spores produced by the fungus inside and over the vineyard. An estimate of the biological parameters in the model suggests to use a singular perturbation analysis. It allows us to compute the speed and the profile of the travelling waves. The analytical results are compared with numerical simulations.
\end{abstract}

Key words: phytopathology, SIR model, travelling waves, singular perturbations AMS subject classification: 92D30, 35K57, 35B20, 35B40

\section{Introduction}

Powdery mildew is one of the most encountered diseases of the vine. Caused by the fungus Uncinula necator, a better knowledge of its mechanisms of propagation would help to devise a better management of the epidemic.

Since Vanderplank [13], a number of models have been proposed for the propagation of plant diseases. Recent papers investigate the role of a dispersal mechanism occurring on two or more spatial scales, see e.g. $[4,7,12]$. In [14] the authors proposed such a model in the context of a fungal disease in order to study the impact of the dual dispersal upon the velocity of an epidemic wave. It is based on partial differential equations of the reaction-diffusion type describing the

\footnotetext{
${ }^{1}$ Corresponding author. E-mail: ducrot@sm.u-bordeaux2.fr
} 
spores dual dispersal coupled with a delay differential equation describing the evolution of the disease.

In the case of a fungal epidemic propagating over a vineyard, a complex model that couples growth of the host and a dual dispersal mechanism for the spores has been devised [3]. Two ranges of dispersal are taken into account in this model. At short range (vinestock scale) spores disperse according to a ray tracing like technique, while at longer range (between vinestocks) a dispersal kernel of gaussian type is used. A crucial question is how to model the longer range dispersal. To investigate this question, a simplified version of this model has been developed in [1]. It is similar to the model considered in [14] but uses a SEIR formulation rather than differential delay equations.

The purpose of this paper is to estimate the spreading rate of the epidemic for a model similar to the one described in [1]. The propagation phenomenon can be described by a travelling wave. The rate of expansion is given by the wave speed. This approach has been extensively used in the study of biological invasions, see e.g. [5, 8, 9, 11].

This paper is organized as follows. In Section 2 we present the model and discuss the values of the parameters. The different time scales involved in the propagation mechanism allow us to perform a singular perturbation analysis. This method provides the asymptotic profile of the solution together with the velocity of propagation (Section 3). The analytical velocity value is compared with numerical simulations of the model (Section 4).

\section{Mathematical model}

In [1] the authors suggested a model for spreading of an epidemic of powdery mildew over a vineyard. We investigate a simplified version of this model in which we neglect growth of the host and we focus on the asymptotic behaviour of solutions. Moreover, for the sake of simplicity we do not take into account a latent phase. This case will be studied in an upcoming article.

We consider a SIR model coupled with reaction-diffusion equations. The vectors of the epidemic are the spores produced by the colonies of fungus located at the vegetal tissue, mostly leaves. Assuming that the time variation of a colony size can be neglected, we choose the infection unit to be a colony as in [10]. For the host, this infection unit is a site that may be occupied by a colony of fungus or not.

The life cycle of the pathogen is the following: when spores land upon a free colony site, they may germinate and create a new colony of fungus which will produce spores in their turn during some sporulating period.

Let $H(t, x)$ denote the spatial density of susceptible (healthy) sites at time $t$ and position $x$, $I(t, x)$ the spatial density of infected (sporulating) sites and $R(t, x)$ the density of removed sites (post-sporulating). The total spatial density of sites $N_{0}=H+I+R$ is assumed to be constant with respect to space and time.

We take into account two ranges for dispersal: short range (spores disperse inside the vine stock where they originate), and a longer range (spores disperse at the vineyard scale). Let $S(t, x)$ denote the density of spores produced by the colonies. The spores total density $S$ is subdivided 
Table 1: Model parameters

\begin{tabular}{lll}
\hline parameter & description & value \\
\hline$\delta$ & deposition rate & $50 \mathrm{day}^{-1}$ \\
$D_{1}$ & short range diffusion coefficient & $50 \mathrm{~m}^{2}$ day $^{-1}$ \\
$D_{2}$ & long range diffusion coefficient & $5000 \mathrm{~m}^{2}$ day $^{-1}$ \\
$r$ & spores production rate & $10^{4}$ spores day $^{-1}{\text { colony } \text { site }^{-1}}$ \\
$F$ & short range vs. long range dispersion & 0.8 \\
$E$ & inoculum effectiveness & $0.1 \%$ \\
$p$ & infectious period duration & 10 days \\
$N_{0}$ & total density of colony sites & $40 \mathrm{~m}^{-2}$ colony sites \\
\hline
\end{tabular}

according to the range of dispersal, short range dispersal spores density $S_{1}$ and longer range spores $S_{2}$. They are produced by a sporulating colony with the rate $r>0$ and may disperse at short range with a constant probability $F \in[0,1]$ and at longer range with probability $(1-F)$.

We assume that spores spread according to a Fickian diffusion coefficient $D_{1}>0$ (short range) and $D_{2}>D_{1}>0$ (longer range) as in [14]. Spores fall down upon the host with some deposition rate $\delta>0$.

These assumptions lead to the following equations for spreading of spores :

$$
\begin{gathered}
\frac{\partial S_{1}}{\partial t}=D_{1} \Delta S_{1}-\delta S_{1}+r F I \\
\frac{\partial S_{2}}{\partial t}=D_{2} \Delta S_{2}-\delta S_{2}+r(1-F) I
\end{gathered}
$$

We assume that the contamination of the sites by spores is described by a proportionate mixing law. It is in accordance with the underlying hypothesis of classical epidemiologic models in phytopathology (see Vanderplank [13]) that the rate of increase of diseased tissue is proportional to the amount of spores multiplied by the probability that these spores fall down on healthy tissues. We have:

$$
\begin{gathered}
\frac{\partial H}{\partial t}=-E \delta\left(S_{1}+S_{2}\right) \frac{H}{N_{0}}, \\
\frac{\partial I}{\partial t}=E \delta\left(S_{1}+S_{2}\right) \frac{H}{N_{0}}-\frac{1}{p} I, \\
\frac{\partial R}{\partial t}=\frac{1}{p} I,
\end{gathered}
$$

where $E$ is the inoculum effectiveness and $p$ the duration of the infective phase.

The parameters of the model and their values are given in Table 1.

Let us explain how the values of the dispersal parameters $\delta$ and $D_{i}$ are estimated. All spores lifted up in the atmosphere fall down within half an hour so the deposition rate $\delta$ is approximately 50 day $^{-1}$. 
To estimate the diffusion coefficients $D_{1}$ and $D_{2}$, we only consider the spores dispersal mechanism. Let $D$ be a diffusion coefficient and $\delta$ be a deposition rate. The density $S$ of spores dispersed in the atmosphere and produced by a single source obeys the following equation:

$$
\left\{\begin{array}{l}
\frac{\partial S}{\partial t}(t, x)=D \Delta S(t, x)-\delta S(t, x), \forall(t, x) \in \mathbb{R}^{2} \times \mathbb{R}^{+} \\
S(x, 0)=\delta_{0}(x), \forall x \in \mathbb{R}^{2}
\end{array}\right.
$$

where $\delta_{0}(x)$ is the Dirac function. Then the total amount of fallen spores upon the vineyard at some point $x \in \mathbb{R}^{2}$ is

$$
P(x)=\int_{0}^{+\infty} \delta S(t, x) d t
$$

where $P(x)$ is the probability density of fallen spores. It can be explicitly computed and its variance is $\sigma=\sqrt{D / \delta}$. The values of $D_{1}$ and $D_{2}$ in Table 1 have been chosen so that $\sigma=1 \mathrm{~m}$ for the short range dispersal and $\sigma=10 \mathrm{~m}$ for the long range dispersal.

The basic reproductive rate of the disease is

$$
R_{0}=r p E
$$

Using the Routh-Hurwitz criterium, it can be easily computed by investigating the linear stability of the trivial equilibrium point $\left(S_{1}, S_{2}, H, I, R\right)=\left(0,0, N_{0}, 0,0\right)$ of system (2..1)-(2..5).

From a biological point of view $R_{0}$ is the number of secondary colonies produced by one infected colony. Indeed, $R_{0}$ equals the number of spores $r$ produced by a single colony during the infectious period $p$ multiplied by the probability that these spores create a new colony.

We perform a parameter reduction for the model by using the dimensionless variables:

$$
\begin{gathered}
\tilde{S}_{1}=\frac{\delta S_{1}}{r N_{0}}, \quad \tilde{S}_{2}=\frac{\delta S_{2}}{r N_{0}}, \quad \tilde{H}=\frac{H}{N_{0}}, \quad \tilde{I}=\frac{I}{N_{0}}, \\
\tilde{t}=\frac{t}{p}, \quad \tilde{x}=\frac{x}{\sqrt{p D_{1}}}, \quad \eta=p \delta, \quad d=\frac{D_{2}}{D_{1}} .
\end{gathered}
$$

Omitting the tilde and equation (2..5), which is not used to solve the problem, we obtain the following system

$$
\begin{gathered}
\frac{\partial S_{1}}{\partial t}=\Delta S_{1}-\eta S_{1}+\eta F I, \\
\frac{\partial S_{2}}{\partial t}=d \Delta S_{2}-\eta S_{2}+\eta(1-F) I, \\
\frac{\partial H}{\partial t}=-R_{0}\left(S_{1}+S_{2}\right) H, \\
\frac{\partial I}{\partial t}=R_{0}\left(S_{1}+S_{2}\right) H-I .
\end{gathered}
$$

The parameter $\eta$ equals the ratio of the time scale of the life cycle of the pathogen over the time scale of the spores dispersal. From the biological parameters listed in Table 1, we can see that 
$\eta=500$ is a large parameter since the dispersal process is fast compared with the duration of the sporulating period.

Similarly the basic reproductive rate $R_{0}$ equals 10 . Thus we may assume that $R_{0}$ is of the order $O(\sqrt{\eta})$ and we write $R_{0}=r_{0} \sqrt{\eta}$ with $r_{0}=O(1)$. Finally we consider the system

$$
\begin{gathered}
\frac{\partial S_{1}}{\partial t}=\Delta S_{1}-\eta S_{1}+\eta F I, \\
\frac{\partial S_{2}}{\partial t}=d \Delta S_{2}-\eta S_{2}+\eta(1-F) I, \\
\frac{\partial H}{\partial t}=-r_{0} \sqrt{\eta}\left(S_{1}+S_{2}\right) H, \\
\frac{\partial I}{\partial t}=r_{0} \sqrt{\eta}\left(S_{1}+S_{2}\right) H-I .
\end{gathered}
$$

Both from biological observations and numerical simulations (see thereafter), this propagation arises due to travelling waves. In order to investigate these travelling waves we use a singular perturbation analysis. This method allows us to derive an expression for the wave speed and also gives us information about the asymptotic profiles of the solution.

\section{Propagation analysis: travelling waves}

In this section, we study system (2..11)-(2..14) considered for nonnegative time $t$ and on the real line $x \in \mathbb{R}$. We are interested in travelling wave solutions, that is particular solutions of the form

$$
\left(S_{1}, S_{2}, H, I\right)(t, x)=\left(\tilde{S}_{1}, \tilde{S}_{2}, \tilde{H}, \tilde{I}\right)(z),
$$

where $z=x-c t$ and $c$ is the wave speed, an unknown positive real number that has to be found together with the functions $\left(\tilde{S}_{1}, \tilde{S}_{2}, \tilde{H}, \tilde{I}\right)$. To simplify the notations we omit the tilde and we are concerned with the following system of ordinary differential equations (here ' denotes the derivative with respect to the variable $z$ ):

$$
\begin{gathered}
-c S_{1}^{\prime}=S_{1}^{\prime \prime}-\eta S_{1}+\eta F I, \\
-c S_{2}^{\prime}=d S_{2}^{\prime \prime}-\eta S_{2}+\eta(1-F) I, \\
c H^{\prime}=r_{0} \sqrt{\eta} H\left(S_{1}+S_{2}\right), \\
-c I^{\prime}=r_{0} \sqrt{\eta} H\left(S_{1}+S_{2}\right)-I,
\end{gathered}
$$

with the following behavior at infinity:

$$
\left(S_{1}, S_{2}, H, I\right)(-\infty)=(0,0,0,0), \quad\left(S_{1}, S_{2}, H, I\right)(+\infty)=(0,0,1,0) .
$$

This means that the solution connects the disease free state $(z=+\infty)$ to the post-infected state $(z=-\infty)$. The variables that describe the disease, that is $S_{1}, S_{2}$ and $I$, are pulse-like functions. 
Here in the limit $\eta \rightarrow+\infty$, the profile of the solution can be split into three different regions: the region where the contamination has not yet begun (ahead of the front), the region where it is over (behind the front) and a thin layer where the contamination takes place (the reaction zone). A dimension analysis of the reaction layer shows that its length is of the order $O\left(\frac{1}{\eta^{1 / 2}}\right)$. Using formal asymptotic analysis, we obtain the profile of the solution.

We first consider the outer problem and for this purpose we set

$$
\left(S_{1}, S_{2}, H, I\right)(z)=\left(S_{1}^{0}, S_{2}^{0}, H^{0}, I^{0}\right)(z)+\frac{1}{\eta}\left(S_{1}^{1}, S_{2}^{1}, H^{1}, I^{1}\right)(z)+O\left(\frac{1}{\eta^{2}}\right) .
$$

Substituting this expansion into system (3..1)-(3..4), we obtain up to the first order:

$$
\begin{gathered}
S_{1}^{0}=F I^{0}, \quad S_{2}^{0}=(1-F) I^{0}, \\
H^{0}\left(S_{1}^{0}+S_{2}^{0}\right)=0 \\
c I^{0^{\prime}}=I^{0},
\end{gathered}
$$

Finally, using (3..5), we obtain the following outer problem:

for $z>0$ (ahead of the front):

$$
S_{1}=S_{2}=I \equiv 0, \quad H \equiv 1
$$

and for $z<0$ (behind the front):

$$
\begin{aligned}
& H \equiv 0, \quad S_{1}=F I, \quad S_{2}=(1-F) I, \\
& c I^{\prime}=I .
\end{aligned}
$$

Therefore the resolution of this outer problem leads us to the profiles of the solutions:

$$
\begin{aligned}
& H(z)=\mathbb{1}_{z>0}, \quad I(z)=I_{0} e^{z / c} \mathbb{1}_{z<0} \\
& S_{1}(z)=I_{0} F e^{z / c} \mathbb{1}_{z<0}, \quad S_{2}(z)=I_{0}(1-F) e^{z / c} \mathbb{1}_{z<0},
\end{aligned}
$$

where $\mathbb{1}_{z>0}$ is the Heaviside function and $I_{0}$ is some constant that should be determined by matching these expressions to the inner problem.

In order to analyze the inner problem, we introduce a stretched variable $\xi=\eta^{1 / 2} z$ and put

$$
\left(s_{1}, s_{2}, h, i\right)(\xi)=\left(S_{1}, S_{2}, H, I\right)(\sqrt{\eta} z),
$$

then:

$$
\begin{aligned}
& s_{1}^{\prime \prime}+\frac{c}{\sqrt{\eta}} s_{1}^{\prime}-s_{1}+F i=0, \\
& d s_{2}^{\prime \prime}+\frac{c}{\sqrt{\eta}} s_{2}^{\prime}-s_{2}+(1-F) i=0, \\
& c h^{\prime}=r_{0} h\left(s_{1}+s_{2}\right), \\
& c i^{\prime}=-r_{0} h\left(s_{1}+s_{2}\right)+\frac{1}{\sqrt{\eta}} i .
\end{aligned}
$$


If we expand the vector function $\left(s_{1}, s_{2}, h, i\right)$ in the powers of $1 / \eta$ :

$$
\left(s_{1}, s_{2}, h, i\right)(\xi)=\left(s_{1}^{0}, s_{2}^{0}, h^{0}, i^{0}\right)(\xi)+\frac{1}{\eta}\left(s_{1}^{1}, s_{2}^{1}, h^{1}, i^{1}\right)(\xi)+O\left(\frac{1}{\eta^{2}}\right),
$$

and plug this expansion into (3..13), we obtain for the zero order terms:

$$
\begin{aligned}
& \left(s_{1}^{0}\right)^{\prime \prime}-s_{1}^{0}+F i^{0}=0 \\
& d\left(s_{2}^{0}\right)^{\prime \prime}-s_{2}^{0}+(1-F) i^{0}=0 \\
& c\left(h^{0}\right)^{\prime}=r_{0} h^{0}\left(s_{1}^{0}+s_{2}^{0}\right) \\
& c\left(i^{0}\right)^{\prime}=-r_{0} h^{0}\left(s_{1}^{0}+s_{2}^{0}\right) .
\end{aligned}
$$

From the matching conditions, we have:

$$
\left(s_{1}^{0}, s_{2}^{0}, h^{0}, i^{0}\right)( \pm \infty)=\left(S_{1}^{0}, S_{2}^{0}, H^{0}, I^{0}\right)\left(0^{ \pm}\right) .
$$

Taking the sum of the last two equations in (3..15) we obtain that $h^{0}+i^{0}$ is a constant function. Since $\left(S_{1}^{0}, S_{2}^{0}, H^{0}, I^{0}\right)\left(0^{+}\right)=(0,0,1,0)$, then it follows from the matching conditions $(3 . .16)$ that $h^{0}(\xi)+i^{0}(\xi) \equiv 1$. Since $H^{0}\left(0^{-}\right)=h^{0}(-\infty)=0$, then $i^{0}(-\infty)=I^{0}\left(0^{-}\right)=1$. This equality allows us to determine the unknown constant $I_{0}$ in (3..12): $I_{0}=1$.

Finally we obtain the following explicit expression for the solution of the outer problem:

$$
\begin{aligned}
& H(z)=\mathbb{1}_{z>0}, \quad I(z)=e^{z / c} \mathbb{1}_{z<0} \\
& S_{1}(z)=F e^{z / c} \mathbb{1}_{z<0}, \quad S_{2}(z)=(1-F) e^{z / c} \mathbb{1}_{z<0} .
\end{aligned}
$$

The matching conditions do not allow us to determine the wave speed. It can be found from the inner problem. Its solutions should match the outer solution and should also be positive in order to ensure the positivity of the travelling wave. The complete inner problem consists of system (3..15) together with the following limits at infinity (superscript 0 is omitted)

$$
\left(s_{1}, s_{2}, h, i\right)(\xi=-\infty)=(F, 1-F, 0,1), \quad\left(s_{1}, s_{2}, h, i\right)(\xi=+\infty)=(0,0,1,0) .
$$

As already noticed, since $h \equiv 1-i$ this system of four differential equations reduces to a system of three, that reads:

$$
\begin{aligned}
& s_{1}^{\prime \prime}-s_{1}+F i=0, \\
& d s_{2}^{\prime \prime}-s_{2}+(1-F) i=0, \\
& c i^{\prime}=-r_{0}(1-i)\left(s_{1}+s_{2}\right) \\
& \left(s_{1}, s_{2}, i\right)(\xi=-\infty)=(F, 1-F, 1), \quad\left(s_{1}, s_{2}, i\right)(\xi=+\infty)=(0,0,0) .
\end{aligned}
$$

We first investigate conditions that ensure the positivity of the solution in the general case $0<$ $F<1$. For that purpose we assume that the solution has an exponential behaviour at $\xi=+\infty$ :

$$
\left(s_{1}, s_{2}, i\right)(\xi) \approx e^{\lambda \xi}\left(\sigma_{1}, \sigma_{2}, \kappa\right), \quad \xi \rightarrow+\infty
$$


The positivity of the solution relies on the positivity of the components of the vector $\mathbf{V}=\left(\sigma_{1}, \sigma_{2}, \kappa\right)$ and $\lambda$ should be a non positive real number. Putting this asymptotic into (3..18), we obtain the following system of equations for $\lambda$ and $\mathbf{V}$ :

$$
\begin{aligned}
& \left(\lambda^{2}-1\right) \sigma_{1}+F \kappa=0, \\
& \left(d \lambda^{2}-1\right) \sigma_{2}+(1-F) \kappa=0, \\
& c \lambda \kappa=-r_{0}\left(\sigma_{1}+\sigma_{2}\right) .
\end{aligned}
$$

Since $\mathbf{V}$ is a positive vector, $F \in(0,1)$ and $\lambda$ is a non positive real number we obtain from the two first equations that $\lambda$ has to be in the interval $(\max (-1,-1 / \sqrt{d}), 0)$. Therefore, since $d$ is larger than one, we obtain the following necessary condition: the equation

$$
\operatorname{det}\left(\begin{array}{ccc}
\lambda^{2}-1 & 0 & F \\
0 & d \lambda^{2}-1 & 1-F \\
r_{0} & r_{0} & c \lambda
\end{array}\right)=0
$$

has a real solution in the interval $\left(-\frac{1}{\sqrt{d}}, 0\right)$.

This condition re-writes as follows: there exists $\lambda \in\left(-\frac{1}{\sqrt{d}}, 0\right)$ such that

$$
\frac{r_{0}}{c}=\frac{(-\lambda)\left(1-\lambda^{2}\right)\left(1-d \lambda^{2}\right)}{(1-F)\left(1-\lambda^{2}\right)+F\left(1-d \lambda^{2}\right)}:=\Phi(\lambda, F, d),
$$

this implies that

$$
\frac{r_{0}}{c} \leq M^{*}(F, d), \quad \text { where } M^{*}(F, d)=\sup _{\lambda \in\left(-\frac{1}{\sqrt{d}}, 0\right)} \Phi(\lambda, F, d)>0 .
$$

This yields a lower bound of the wave speed for $0<F<1$ :

$$
c \geq c^{*}(F, d) \text { with } c^{*}(F, d)=\frac{r_{0}}{M^{*}(F, d)} .
$$

We now consider the degenerate situation $F=1$ (short dispersion only). In this case system $(3 . .18)$ reduces to the system of two equations $\left(s_{2} \equiv 0\right)$ :

$$
\begin{aligned}
& s_{1}^{\prime \prime}-s_{1}+i=0, \\
& c i^{\prime}=-r_{0}(1-i) s_{1} \\
& \left(s_{1}, i\right)(\xi=-\infty)=(1,1), \quad\left(s_{1}, i\right)(\xi=+\infty)=(0,0) .
\end{aligned}
$$

As before we suppose that the solution has an exponential behavior at $\xi=+\infty$ :

$$
\left(s_{1}, i\right)(\xi) \approx e^{\lambda \xi}\left(\sigma_{1}, \kappa\right), \quad \xi \rightarrow+\infty .
$$

Using the same argument as in the general case we obtain the following condition: there exists $\lambda \in(-1,0)$ such that

$$
\frac{r_{0}}{c}=-\lambda\left(1-\lambda^{2}\right)
$$


This condition re-writes as follows:

$$
c \geq \frac{3 \sqrt{3}}{2} r_{0} .
$$

The degenerate case $F=0$ can be investigated in the same way and we find

$$
c \geq \frac{3 \sqrt{3 d}}{2} r_{0}
$$

Finally we have found the following lower bound for the wave speed:

$$
c \geq\left\{\begin{array}{l}
\frac{3 \sqrt{3 d}}{2} r_{0} \text { for } F=0 \\
c^{*}(F, d) \text { given in }(3 . .22) \text { for } 0<F<1 \\
\frac{3 \sqrt{3}}{2} r_{0} \text { for } F=1
\end{array}\right.
$$

Here are some qualitative properties of the minimal wave speed $c^{*}(F, d)$, see Appendix A for the proof. It is increasing with respect to $d$ and decreasing with respect to $F$. In addition when $d$ is large we have the following asymptotic:

$$
c^{*}(F, d) \approx r_{0} \sqrt{d} \frac{1-F x(F)}{\sqrt{x(F)}(1-x(F))}, \text { for } d \rightarrow+\infty,
$$

where $x(F)$ is defined by

$$
x(F)=\frac{1}{2 F}(3-F-\sqrt{(1-F)(9-F)}) .
$$

We will numerically show in the next section that this necessary condition on the wave speed is a sufficient condition also and that the quantity in the right hand side of (3..24) corresponds to the spreading rate of the epidemic.

Remark 3..1. Using (3..22) we can notice that if $d$ is large enough, we have

$$
\frac{3 \sqrt{3 d}}{2} r_{0}=\lim _{F \rightarrow 0^{+}} c^{*}(F, d), \text { and } \frac{3 \sqrt{3}}{2} r_{0} \neq \lim _{F \rightarrow 1^{-}} c^{*}(F, d)=r_{0} \sqrt{d} \frac{d}{d-1} .
$$

Therefore the minimal wave speed is discontinuous at $F=1$ and continuous at $F=0$. This remark will be confirmed in the next section from comparison with numerical simulations.

\section{Numerical simulations}

In this section we compare the numerical simulations with the asymptotic formulas that have been obtained above. The numerical simulations of system (2..11)-(2..14) are fulfilled by using a semiimplicit finite difference discretization for the reaction-diffusion equations and Euler scheme for 
the ordinary differential equations. The values of the parameters for numerical simulations are taken from the biological observations (cf. Table 1):

$$
\eta=500, \quad d=100, \quad r_{0}=\frac{10}{\sqrt{500}} .
$$

Fig. 1 shows the solution for the case $F=0.8$. We can see that the reaction occurs in a very thin layer and the components of the solution are close to the analytical profiles obtained above (see (3..17)) from the asymptotic analysis of the limiting problem $(\eta \rightarrow+\infty)$. In particular, $S_{1} \approx F I$ and $S_{2} \approx(1-F) I$.
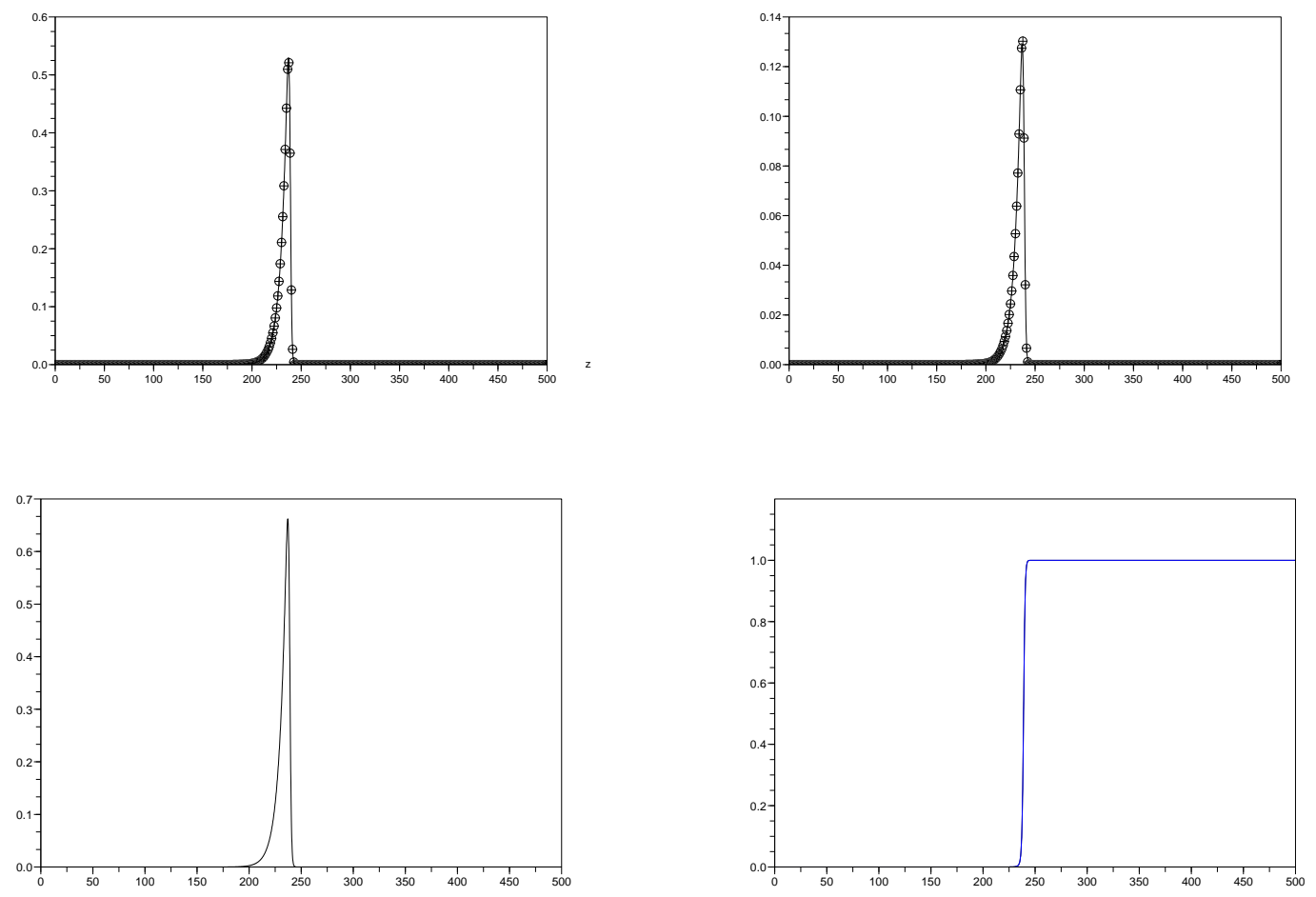

Fig. 1: Travelling wave solution: $S 1$ (continuous line) and $F I$ (dots) (top left), $S 2$ (continuous line) and $(1-F) I$ (dots)(top right), $I$ (bottom left) and $H$ (bottom right).

Next we vary the value of $F$ in order to study its influence on the wave speed. Fig. 2 represents a comparison of the numerical wave speeds with those obtained from the asymptotic analysis (3..24). We first observe that the numerical wave speed and the analytical one differ from each other for less than $5 \%$, except for the cases corresponding to a single dispersion ( $F=1$, short dispersion and $F=0$, long dispersion), where for these limit situations the wave speed error is about $8 \%$.

Next we observe that the introduction of a long dispersion (even with a very little proportion $F<1$ ) generates a rapid jump of the wave speed. The wave speed is discontinuous at $F=1$ when long dispersion is introduced. This observation confirms the analytical expression for the minimal wave speed (see Remark 3.1). In other words it seems that the travelling wave corresponding to 
a single short dispersion is unstable with respect to perturbations of long dispersion type. Such a phenomenon has already been noticed by several authors (see for example [12] and the references therein).

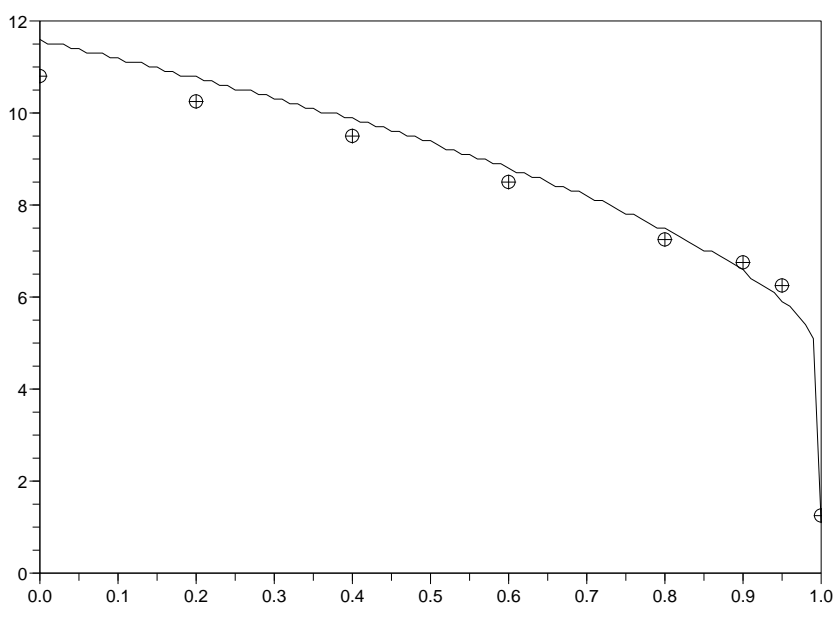

Fig. 2: Comparison of the analytical and numerical (dots) values of the wave speed for different values of $F$.

\section{Concluding remarks}

In this work we have studied the influence of a dual dispersal mechanism on the rate of invasion of a powdery mildew epidemic over a vineyard. The model we use is based on a reaction-diffusion system coupled with ordinary differential equations.

We have obtained that the wave speed is discontinuous with respect to the proportion of long range dispersal and more specifically at $F=1$ that corresponds to the case of short range dispersal only. This result is quite similar to the ones obtained for various models in the literature: on the one hand, in the context of composite dispersal kernels for integral kernel based models (see [7, 12]), and on the other hand, for a simple model of a reaction-diffusion equation coupled with an ordinary differential one (see [6]).

However for all these models the wave speed is monotonic with respect to the proportion of long dispersal (cf. fig. 2). Nevertheless a non monotonic behavior of the wave speed has been observed in [14] for the model with delay differential equations: a certain proportion of long distance dispersal leads to an optimal velocity. Delay reaction-diffusion equations seem to exhibit a more complex behavior.

Now let us discuss the biological values that were obtained with our model: we found an asymptotic rate of expansion $v_{b} \approx c \sqrt{D_{1} / p} \approx 2$ m.day ${ }^{-1}$ for short range dispersal only ( $F=$ 1 ), and for $F<1, v_{b}$ lies between 10 and 25 m.day ${ }^{-1}$. From biological observations the rate of expansion of powdery mildew epidemic is of order $1 \mathrm{~m}$. day $^{-1}$. Though the spreading rate 
computed is too high, it is not absurd considering the model is quite simple. For instance we did not take into account the row structure of vineyards that is likely to slow the disease. This situation will be investigated in a forthcoming paper.

\section{Appendix. Qualitative properties of the minimal wave speed}

In this Appendix we prove the qualitative properties of the minimal wave speed $c^{*}(F, d)$ introduced in Section 3. Let us first notice that the quantity $M^{*}(F, d)$ defined in (3..21) can be re-written as:

$$
M^{*}(F, d)=\frac{1}{\sqrt{d}} \sup _{x \in(0,1)} \frac{x\left(1-x^{2}\right)\left(1-\frac{x^{2}}{d}\right)}{(1-F)\left(1-\frac{x^{2}}{d}\right)+F\left(1-x^{2}\right)} .
$$

We set the function

$$
\phi(x, F, \epsilon)=\frac{x\left(1-x^{2}\right)\left(1-\epsilon x^{2}\right)}{(1-F)\left(1-\epsilon x^{2}\right)+F\left(1-x^{2}\right)},
$$

and the quantity $M^{*}(F, d)$ re-writes

$$
M^{*}(F, d)=\frac{1}{\sqrt{d}} \sup _{(0,1)} \phi\left(., F, \frac{1}{d}\right) .
$$

If we compute the partial derivatives of function $\phi$ with respect to $\epsilon$ and $F$ we obtain:

$$
\begin{gathered}
\frac{\partial \phi}{\partial \epsilon}=\frac{F x^{5}\left(1-x^{2}\right)}{\left((1-F)\left(1-\epsilon x^{2}\right)+F\left(1-x^{2}\right)\right)^{2}} \geq 0, \text { for any } x \in(0,1), \\
\frac{\partial \phi}{\partial F}=\frac{x^{2}(1-\epsilon)}{\left((1-F)\left(1-\epsilon x^{2}\right)+F\left(1-x^{2}\right)\right)^{2}} \geq 0, \text { for any } 0<\epsilon<1 .
\end{gathered}
$$

Therefore $M^{*}(F, d)$ is increasing with respect to $F$ and decreasing with respect to $d$. Finally from (3..22) we obtain that $c^{*}(F, d)$ is decreasing with respect to $F$ and increasing with respect to $d$.

We now consider the case where $d$ is large $(d>>1)$. Then we have

$$
\phi\left(x, F, \frac{1}{d}\right) \approx \phi(x, F, 0) \approx \frac{x\left(1-x^{2}\right)}{(1-F)+F\left(1-x^{2}\right)} \text { when } d \rightarrow+\infty .
$$

Therefore we obtain that

$$
M^{*}(F, d) \approx \frac{1}{\sqrt{d}} \sup _{(0,1)} \phi(., F, 0),
$$

and from (3..22) we conclude that

$$
c^{*}(F, d) \approx r_{0} \sqrt{d} \frac{1}{\sup _{(0,1)} \phi(., F, 0)} .
$$


It remains to compute the supremum in the above formula.

For that purpose let us introduce the function $\psi$ defined by

$$
\psi(x)=\frac{1-F x^{2}}{x\left(1-x^{2}\right)} .
$$

Simple computations show that the derivative of this function vanishes at $x$ satisfying the polynomial equation:

$$
F x^{4}-(3-F) x^{2}+1=0 .
$$

Since $F$ lies in $(0,1)$ we see that this equation has exactly two positive real roots $x^{ \pm}$given by

$$
\left(x^{ \pm}\right)^{2}=\frac{1}{2 F}(3-F \pm \sqrt{(1-F)(9-F)}) .
$$

In addition we easily see that $\left(x^{-}\right)^{2} \in(0,1)$ while $\left(x^{+}\right)^{2}>1$. Therefore we conclude that:

$$
\frac{1}{\sup _{(0,1)} \phi(., F, 0)}=\inf _{(0,1)} \psi=\psi\left(x^{-}\right)
$$

that completes the proof of (3..25).

\section{References}

[1] J.-B. Burie, A. Calonnec, M. Langlais. Modeling of the invasion of a fungal disease over a vineyard. Proceedings of ECMTB05, in press.

[2] A. Calonnec, P. Cartolarao, L. Deliere, J. Chadoeuf. Powdery mildew on grapevine: effect of the date of primary contamination on the disease development on leaves and the damages on grape. Proceedings of Organisation for biological and integrated control of noxious animals and plants Workshop, Brescia, Italy, in press.

[3] A. Calonnec, G. Latu, J. Naulin, J. Roman, G. Tessier. Parallel simulation of the propagation of powdery mildew in a vineyard. Proceedings of Euro-Par 2005, Lecture Notes in Computer Science, 3648, (2005) 1254-1264.

[4] J.S. Clark, C. Fastie, G. Hurtt, et al. Reid's paradox of rapid plant migration. Bioscience, 48 (1998), 13-24.

[5] P. Fife. Mathematical aspects of reacting and diffusing systems. Lecture Notes in Biomathematics, 28, Springer Verlag, Berlin, 1979.

[6] L. Goldwasser, J. Cook, E.D. Silverman. The effects of variability on metapopulation dynamics and rates of invasions. Ecology, 75 (1994), 40-47. 
[7] J.A. Metz, D. Mollison, F. van den Bosch. The geometry of ecological interactions simplifying spatial complexity. In: U. Diekmann, J.A.J. Metz, (eds). The Dynamics of Invasion Waves. Cambridge Studies in Adaptive Dynamics. pp. 482-512, Cambridge University Press, Cambridge, 2000.

[8] J.D. Murray. Mathematical Biology. II. Spatial Models and Biomedical Applications, 3rd edition. Springer Verlag, Berlin, 2003.

[9] A. Okubo, S.A. Levin. Diffusion and ecological problems: Modern perspectives, 2nd edition. Springer-Verlag, New York, 2001.

[10] J. Segarra, M.J. Seger, F. van den Bosch. Epidemic dynamics and patterns of plant diseases. Phytopathology, 91 (2001), 1001-1010.

[11] N. Shigesada, K. Kawasaki. Biological invasions: Theory and practice. Oxford Series in Ecology and Evolution. Oxford University Press, Oxford, 1997.

[12] N. Shigesada, K. Kawasaki. Invasion and the range expansion of species: effects of longdistance dispersal. In: J. Bullock, R. Kenward, R. Hails (eds). Dispersal Ecology, 350-373. Blackwell Science, Malden MA, 2002.

[13] J.E. Vanderplank. Plant diseases: epidemics and control. Academic Press, New York, 1963.

[14] M.W. Zawolek, J.C. Zadoks. Studies in focus development: an optimum for the dual dispersal of plant pathogens. Phytopathology, 82 (1992), 1288-1297. 